\title{
Orthopteroid insects (Mantodea, Blattodea, Dermaptera, Phasmoptera, Orthoptera) of agrocenosis of rice fields in Kyzylorda oblast, South Kazakhstan
}

\author{
Izbasar I. Temreshev', Arman M. Makezhanov' \\ I LLP «Educational Research Scientific and Production Center "Bayserke-Agro"», Almaty oblast, Pan- \\ filov district, Arkabay village, Otegen Batyr street, 3, Kazakhstan \\ Corresponding author: Izbasar I. Temreshev (temreshev76@mail.ru) \\ Academic editor: R. Yakovlev | Received 10 March 2020 | Accepted 12 April 2020 | Published 16 September 2020 \\ http://zoobank.org/EF2D6677-74E1-4297-9A18-81336E53FFD6 \\ Citation: Temreshev II, Makezhanov AM (2020) Orthopteroid insects (Mantodea, Blattodea, Dermaptera, \\ Phasmoptera, Orthoptera) of agrocenosis of rice fields in Kyzylorda oblast, South Kazakhstan. Acta Biologica \\ Sibirica 6: 229-247. https://doi.org/10.3897/abs.6.e54139
}

\begin{abstract}
An annotated list of Orthopteroidea of rise paddy fields in Kyzylorda oblast in South Kazakhstan is given. A total of 60 species of orthopteroid insects were identified, belonging to 58 genera from 17 families and 5 orders. Mantids are represented by 3 families, 6 genera and 6 species; cockroaches - by 2 families, 2 genera and 2 species; earwigs - by 3 families, 3 genera and 3 species; sticks insects - by 1 family, 1 genus and 1 species. Orthopterans are most numerous ( 8 families, 46 genera and 48 species). Of these, three species, Bolivaria brachyptera, Hierodula tenuidentata and Ceraeocercus fuscipennis, are listed in the Red Book of the Republic of Kazakhstan. Celes variabilis and Chrysochraon dispar indicated for the first time for a given location. The fauna of orthopteroid insects in the studied areas of Kyzylorda is compared with other regions of Kazakhstan.
\end{abstract}

\section{Keywords}

Orthopteroidea, fauna, annotated list, paddy fields, Kyzylorda area

Copyright Izbasar I. Temreshev, Arman M. Makezhanov. This is an open access article distributed under the terms of the Creative Commons Attribution License (CC BY 4.0), which permits unrestricted use, distribution, and reproduction in any medium, provided the original author and source are credited. 


\section{Introduction}

The role of orthopteroid insects in both anthropogenic and various natural ecosystems is difficult to overestimate. This group is represented by both abundant herbivores, periodically causing significant economic damage, and entomophages, exterminating pests, and sapronecrophages, which have important sanitary and epidemiological significance. Thus, the elucidation of their species composition in certain areas, including and agrocenosis and adjacent territories, is a very urgent task. Some information on the fauna of this group in the agrocenosis of paddy fields of rice in the south of Kazakhstan as a whole, and in the Kyzylorda area in particular, is contained in the works of G.G. Jacobson and V.L. Bianchi, G.Ya. Bey-Bienko, F.N. Pravdin, L.A. Kotlyarova, I.A. Komissarova, A.V. Latchininsky and M.G. Sergeev et al., E.O. Shcherbakov and V.Yu. Savitsky (Jacobson and Bianchi 1905; Bey-Bienko 1936, 1950, 1964a, b, c; Komissarova 1973; Kotlyarova 1975; Pravdin 1978; Latchininsky et al. 2002; Shcherbakov and Savitsky 2015; Sergeev et al. 2016), however, completely all groups of orthopteroids living here were not studied. It should be noted that rice paddy fields in the considered area in crop rotation alternate with other crops, most often with alfalfa. Since the fields are nearby, insects often move from alfalfa to rice, and vice versa.

In view of the above, the study of the fauna of orthopteroid insects in the agrocenosis of rice fields in the Kyzylorda oblast is of interest both scientifically and in practical terms.

\section{Material and methods}

The collection of material was carried out in 2018-2019 in the Kyzylorda oblast in southern Kazakhstan during the implementation of the 019 budget programs "Introduction of a domestic biological product based on an entomopathogenic fungus against grasshoppers in the Kyzylorda oblast" in the AkZhol peasant farm in

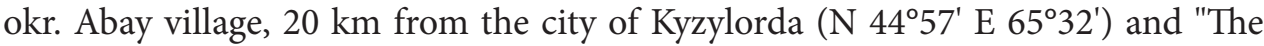
introduction on the basis of a pilot farm in the Kyzylorda oblast of a complex of innovative agricultural technologies with elements of precision farming to increase the productivity of cultivated crops" in the peasant Akmaya farm in the Shieli district, $120 \mathrm{~km}$ from the city of Kyzylorda ( $\left.44^{\circ} 9^{\prime} \mathrm{E} 66^{\circ} 56^{\prime}\right)$. When collecting the material, standard entomological methods were used - light trapping, ground net mowing, soil traps, manual collection. The larvae of some species of grasshoppers and locusts were collected alive and then grown in cages to adults. For convenience, the names of the main localities and surnames of the collectors are abbreviated: KO - Kyzylorda oblast, IT - I.I. Temreshev, AM - A.M. Makezhanov. Other abbreviations: L - larva (L3 - larva 3 instar, L4 - larva 4 instar, L5 - larva 5 instar), v. - village, c. - city, neig. - neighborhood, d. - district, PF - peasant farming. The taxonomy of all orders, families and other taxa of all identified orthopteroid insects is given in 
accordance with electronic databases (Beccaloni 2019; Brock et al. 2019; Cigliano et al. 2019; Hopkins et al. 2019; Otte et al. 2019). Identification of species and their life forms, as well as morphometric measurements of collected specimens of locusts were carried out by I.I. Temreshev using appropriate sources from the list of references (Bei-Bienko 1936, 1950, 1964a, b, c; Pravdin 1978; Childebaev and Storozhenko 2001; Lachininsky et al. 2002; Childebaev and Storozhenko 2004; Storozhenko 2004; Shcherbakov and Savitsky 2015; Sergeev et al. 2016; Temreshev 2017). The material is stored in the personal collections of authors.

\section{Results}

Below is an annotated list of species indicating the orthopteroid insect, the collected material, type of food and life form, identified during the study on agrocenosis of rice crops in the Kyzylorda oblast.

\section{Order Mantodea (Mantoptera)}

\section{Family Empusidae}

\section{Empusa pennicornis (Pallas, 1773)}

Material examined. 1 female, 13.07.2018, KO, Shieli d., PF Akmaya, at the hotel, to the light, IT; 2 L, 25.05.2018, KO, neig. v. Abay, PF Akzhol, rice field edge, IT; 1 male, 1 female, 24.06.2019, KO, Shieli d., PF Akmaya, edge of the rice field and alfalfa field, IT.

\section{Family Mantidae}

Bolivaria brachyptera (Pallas, 1773)

Material examined. 1 L, 25.05.2018, KO, neig. v. Abay, PF Akzhol, rice field edge, IT; 1 female, 12.07.2018, KO, Shieli d., PF Akmaya, alfalfa field, IT; 1 male, 24.06.2019, KO, Shieli d., PF Akmaya, alfalfa field, IT.

Conservation status. It is entered in the Red Book of the Republic of Kazakhstan.

\section{Hierodula tenuidentata (Saussure, 1869)}

Material examined. 1 ootheca, 2 L, 14.06.2018, KO, Shieli d., PF Akmaya, by the hotel on the willow, IT; 1 male 1, female, 12.07.2018, KO, Shieli d., PF Akmaya, at the hotel, to the light, IT.

Conservation status. It is entered in the Red Book of the Republic of Kazakhstan. 
Mantis religiosa (Linnaeus, 1758)

Material examined. 1 ootheca, 17.05.2018, KO, neig. v. Abay, PF Akzhol, rice field edge, IT; 1 ootheca, 5 L, 24-26.05.2018, KO, neig. v. Abay, PF Akzhol, rice field edge, IT; 2 males 3 females, 13.07.2018, KO, Shieli d., PF Akmaya, rice field edge and alfalfa field, IT, AM; 4 males, 5 females, 22.06.2019, KO, Shieli d., PF Akmaya, rice field edge, IT, AM; 1 male, 1 female, 24.06.2019, KO, Shieli d., PF Akmaya, rice field edge, IT, AM.

Severinia turcomaniae (Saussure, 1872)

Material examined. 1 male, 12.07.2018, KO, Shieli d., PF Akmaya, at the hotel, to the light, IT.

\section{Family Tarachodidae}

\section{Iris polystictica (Fischer von Waldheim, 1846)}

Material examined. 2 males, 13.07.2018, KO, Shieli d., PF Akmaya, at the hotel, to the light, IT; 2 males, 3 females, 24.06.2019, KO, Shieli d., PF Akmaya, rice field edge, IT, AM; 1 male, 29.08.2019, KO, Shieli d., PF Akmaya, at the hotel, to the light, IT; 1 female, 30.08.2019, KO, Shieli d., PF Akmaya, alfalfa field, IT.

\section{Order Blattodea (Blattoptera)}

\section{Family Blattidae}

\section{Shelfordella lateralis (Walker, 1868)}

Material examined. 1 male, 13.07.2018, KO, Shieli d., PF Akmaya, rice field, at the field camp, IT; 1 female, 14.07.2018, in the same place, by the hotel, under the bark of dry Willow, IT.

Note. Sinanthrop species. In the south and southeast of the country can lead a free lifestyle. In recent years, actively settles in the territory of Kazakhstan to the north.

\section{Family Polyphagidae}

\section{Polyphaga saussurei (Dohrn, 1888)}

Material examined. 1 male, 12.07.2018, KO, Shieli d., PF Akmaya, at the hotel, to the light, IT; 1 female, 14.07.2018, in the same place, rice field edge, soil trap, IT, AM.

Note. Facultative sinanthrop species 


\section{Order Dermaptera}

Family Labiduridae

Labidura riparia (Pallas, 1773)

Material examined. 85 L1, 65 L2, 55 L3, 49 L4, 29 L5, 24-26.05.2018, KO, neig. v. Abay, PF Akzhol, rice field edge, IT; 2 males, 12.07.2018, 3 females, KO, Shieli d., PF Akmaya, at the hotel, to the light, IT, AM; 4 males, 5 females, 13.07.2018, KO, Shieli d., PF Akmaya, rice field edge, soil traps, IT, AM; 6 males, 5 females , 14.07.2018, KO, Shieli d., PF Akmaya, rice field edge, soil traps, IT, AM.

Distribution. Cosmopolitan.

\section{Family Forficulidae}

\section{Anechura bipunctata (Fabricius, 1781)}

Material examined. 7 males, 5 females, 24-26.05.2018, KO, neig. v. Abay, PF Akzhol, rice field edge, IT; 1 male, 2 females, 12.07.2018, KO, Shieli d., PF Akmaya, at the hotel, under dry bark Elaeagnus, IT; 1 male, 1 females, 13.07.2018, in the same place, under the bark of dry Willow, IT; 2 males, 1 female, 14.07.2018, KO, Shieli d., PF Akmaya, rice field edge, soil traps, IT, AM.

\section{Family Spongiphoridae}

\section{Labia minor (Linnaeus, 1758)}

Material examined. 1 male, 1 female, 17.05.2018, KO, neig. v. Abay, PF Akzhol, rice field edge, IT; 3 males, 2 females, 12.07.2018, KO, Shieli d., PF Akmaya, at the hotel, to the light, IT.

\section{Order Phasmoptera (Phasmatodea)}

Family Diapheromeridae

Sceptrophasma bituberculatum (Redtenbacher, 1889)

Material examined. 2 L, 17.05.2018, KO, neig. v. Abay, PF Akzhol, rice field edge, IT; 1 female, 14.07.2018, KO, Shieli d., PF Akmaya, rice field edge, soil traps, IT, AM. 


\section{Order Orthoptera}

\section{Suborder Ensifera}

Family Tettigoniidae

\section{Ceraeocercus fuscipennis Uvarov, 1910}

Material examined. 1 male, 2 females, 12.07.2018, KO, Shieli d., PF Akmaya, rice field edge, net catch, hand picking, IT, AM; 1 female, 24.06.2019, KO, Shieli d., PF Akmaya, rice field edge, hand picking, IT.

Conservation status. It is entered in the Red Book of the Republic of Kazakhstan.

\section{Conocephalus fuscus (Fabricius, 1793)}

Material examined. 4 males, 3 females, 12.07.2018, KO, Shieli d., PF Akmaya, rice field edge and alfalfa field, net catch, IT; 5 males, 6 females, 24.06.2019, KO, Shieli d., PF Akmaya, rice field edge, IT, AM.

\section{Decticus verrucivorus (Linnaeus, 1758)}

Material examined. 5 L3, 4 L4, 2 L5, 17.05.2018, KO, neig. v. Abay, PF Akzhol, rice field edge, IT; 85 L1, 5 L3, 4 L4, 8 L5,24-26.05.2018, KO, neig. v. Abay, PF Akzhol, rice field edge, IT; 1 male, 29.08.2019, KO, Shieli d., PF Akmaya, at the hotel, to the light, IT; 4 males, 5 females, 30.08.2019, KO, Shieli d., PF Akmaya, rice field edge and alfalfa field, net catch, IT; 4 males, 7 females, 24.06.2019, KO, Shieli d., PF Akmaya, rice field edge and alfalfa field, net catch, IT, AM.

\section{Glyphonotus thoracicus (Fischer von Waldheim, 1846)}

Material examined. 1 female, 29.08.2019, KO, Shieli d., PF Akmaya, rice field edge, IT; 2 males, 3 females, 24.06.2019, KO, Shieli d., PF Akmaya, alfalafa field, IT, AM; 1 male, 1 female, 30.08.2019, KO, Shieli d., PF Akmaya, alfalafa field, IT.

Distribution. Endemic of Central Asia.

\section{Phaneroptera falcata (Poda, 1761)}

Material examined. 2 males, 1 female, 30.08.2019, KO, Shieli d., PF Akmaya, rice field edge, net catch, IT, AM; 7 males, 5 females, 24.06.2019, KO, Shieli d., PF Akmaya, rice field edge and alfalfa field, net catch, IT, AM. 


\section{Platycleis affinis Fieber, 1853}

Material examined. 2 L3, 7 L4, 5 L5, 17.05.2018, KO, neig. v. Abay, PF Akzhol, rice field edge, IT; 5 L3, 9 L4, 7 L5, 24-26.05.2018, KO, neig. v. Abay, PF Akzhol, rice field edge, IT, AM; 1 male, 2 females, 12.07.2018, KO, Shieli d., PF Akmaya, at the hotel, to the light, IT; 4 males, 3 females, 12.07.2018, KO, Shieli d., PF Akmaya, rice field edge and alfalfa field, net catch, IT, AM; 3 males, 2 females, 30.08.2019, KO, Shieli d., PF Akmaya, rice field edge, net catch, IT, AM; 2 males, 3 females, 24.06.2019, KO, Shieli d., PF Akmaya, rice field edge and alfalfa field, net catch, IT, AM.

\section{Platycleis intermedia (Audinet-Serville, 1838)}

Material examined. 5 L3, 4 L4, 2 L5, 17.05.2018, KO, neig. v. Abay, PF Akzhol, rice field edge, IT; 855 L3, 4 L4, 2 L5, 24-26.05.2018, KO, neig. v. Abay, PF Akzhol, rice field edge, IT, AM; 2 males, 2 females, 12.07.2018, KO, Shieli d., PF Akmaya, at the hotel, to the light, IT; 3 males, 3 females, 12.07.2018, KO, Shieli d., PF Akmaya, rice field edge and alfalfa field, net catch, IT, AM; 1 males, 2 females, 30.08.2019, KO, Shieli d., PF Akmaya, rice field edge, net catch, IT, AM; 4 males, 5 females, 24.06.2019, KO, Shieli d., PF Akmaya, rice field edge and alfalfa field, net catch, IT, AM.

\section{Ruspolia nitidula (Scopoli, 1786)}

Material examined. 4 males, 3 females, 30.08.2019, KO, Shieli d., PF Akmaya, rice field edge, net catch, IT, AM; 3 males, 5 females, 24.06.2019, KO, Shieli d., PF Akmaya, rice field edge and alfalfa field, net catch, IT, AM.

\section{Semenoviana plotnikovi (Uvarov, 1914)}

Material examined. 1 female, 12.07.2018, KO, Shieli d., PF Akmaya, alfalfa field, net catch, IT.

\section{Tettigonia viridissima (Linnaeus, 1758)}

Material examined. 7 L3, 6 L4, 4 L5, 17.05.2018, KO, neig. v. Abay, PF Akzhol, rice field edge, IT; 4 L5 males, 5 L5 females, 24.05.2018, KO, neig. v. Abay, PF Akzhol, rice field edge, IT, AM; 1 male, 12.07.2018, KO, Shieli d., PF Akmaya, alfalfa field, net catch, IT; 2 males, 3 females, 13.07.2018, KO, Shieli d., PF Akmaya, at the hotel, to the light, IT, AM; 8 males, 10 females, 24.06.2019, KO, Shieli d., PF Akmaya, rice field edge and alfalfa field, net catch, IT, AM; 2 males, 2 females, 30.08.2019, KO, Shieli d., PF Akmaya, rice field edge, net catch, IT. 


\section{Tettigonia caudata (Charpentier, 1845)}

Material examined. 6 L3, 5 L4, 8 L5, 17.05.2018, KO, neig. v. Abay, PF Akzhol, rice field edge, IT; 5 L5 males, 7 L5 females, 24.05.2018, KO, neig. v. Abay, PF Akzhol, rice field edge, IT, AM; 3 males, 4 females, 30.08.2019, KO, Shieli d., PF Akmaya, rice field edge and alfalfa field, net catch, IT, AM; 4 males, 5 females, 24.06.2019, KO, Shieli d., PF Akmaya, rice field edge and alfalfa field, net catch, IT, AM.

\section{Family Gryllidae}

\section{Gryllus campestris Linnaeus, 1758}

Material examined. 1 male, 1 female, 17.05.2018, KO, neig. v. Abay, PF Akzhol, rice field edge, IT; 5 males, 5 females, 12.07.2018, KO, Shieli d., PF Akmaya, at the hotel, to the light, IT, AM; 3 males, 6 females, 13.07.2018, KO, Shieli d., PF Akmaya, rice field edge, soil traps, IT, AM; 5 males, 4 females, 24.06.2019, KO, Shieli d., PF Akmaya, rice field edge and alfalfa fields, soil traps, IT, AM.

\section{Gryllus bimaculatus (De Geer, 1773)}

Material examined. 2 males, 3 females, 17.05.2018, KO, neig. v. Abay, PF Akzhol, rice field edge, IT; 2 males, 3 females, 12.07.2018, KO, Shieli d., PF Akmaya, at the hotel, to the light, IT, AM; 1 male, 1 female, 13.07.2018, KO, Shieli d., PF Akmaya, rice field edge, soil traps, IT, AM; 2 males, 1 female, 24.06.2019, KO, Shieli d., PF Akmaya, rice field edge and alfalfa, soil traps, IT, AM.

\section{Melanogryllus desertus (Pallas, 1771)}

Material examined. 3 males, 2 females, 17.05.2018, KO, neig. v. Abay, PF Akzhol, rice field edge, IT; 2 males, 1 female, 12.07.2018, KO, Shieli d., PF Akmaya, at the hotel, to the light, IT; 3 males, 5 females, 14.07.2018, KO, Shieli d., PF Akmaya, alfalfa field edge, soil traps, IT, AM; 4 males, 5 females, 24.06.2019, KO, Shieli d., PF Akmaya, rice field edge and alfalfa fields, soil traps, IT, AM.

\section{Modicogryllus bordigalensis (Latreille, 1804)}

Material examined. 2 males, 1 female, 12.07.2018, KO, Shieli d., PF Akmaya, at the hotel, to the light, IT; 4 males, 5 females, 13.07.2018, KO, Shieli d., PF Akmaya, rice field edge, soil traps, IT, AM; 3 males, 3 females, 24.06.2019, KO, Shieli d., PF Akmaya, rice and alfalfa field edge, soil traps, IT, AM. 


\section{Oecanthus turanicus (Uvarov, 1912)}

Material examined. 1 male, 2 females, 12.07.2018, KO, Shieli d., PF Akmaya, alfalfa field, net catch, IT; 1 male, 1 female, 24.06.2019, KO, Shieli d., PF Akmaya, rice field edge, manual collection, IT.

\section{Pteronemobius heydenii (Fischer, 1853)}

Material examined. 1 male, 1 female, 13.07.2018, KO, Shieli d., PF Akmaya, rice field edge, soil traps, IT, AM; 1 female, 27.05.2018, KO, neig. v. Abay, PF Akzhol, rice field edge, IT; 4 males, 7 females, 14.07.2018, KO, neig. v. Abay, PF Akzhol, rice field edge, soil traps, IT, AM.

\section{Family Gryllotalpidae}

\section{Gryllotalpa unispina Saussure, 1874}

Material examined. 1 female, 17.05.2018, KO, neig. v. Abay, PF Akzhol, rice field edge, soil traps, IT; 4 males, 5 females, 12.07.2018, KO, Shieli d., PF Akmaya, at the hotel, to the light, IT; 6 males, 5 females, 13.07.2018, KO, Shieli d., PF Akmaya, at the hotel, to the light, IT; 1 males, 13.07.2018, KO, Shieli d., PF Akmaya, rice field edge, soil traps, IT, AM; 2 males, 1 female, 24.06.2019, KO, Shieli d., PF Akmaya, rice field edge and alfalfa fields, soil traps, IT, AM.

\section{Suborder Caelifera}

\section{Family Tridactylidae}

\section{Xya variegata (Latreille, 1809)}

Material examined. 1 male, 1 female, 17.05.2018, KO, neig. v. Abay, PF Akzhol, rice field edge, manual collect, IT; 2 males, 3 females, 12.07.2018, KO, Shieli d., PF Akmaya, rice field edge, soil traps, IT; 1 male, 1 female, 24.06.2019, KO, Shieli d., PF Akmaya, rice field edge and alfalfa fields, soil traps, IT.

\section{Family Pyrgomorphidae}

\section{Pyrgomorpha bispinosa deserti Bey-Bienko \& Mistshenko, 1951}

Material examined. 7 males, 9 females, 17.05.2018, KO, neig. v. Abay, PF Akzhol, rice field edge, IT; 9 males, 12 females, 24-26.05.2018, KO, neig. v. Abay, PF Akzhol, rice field edge, IT; 2 males, 1 female, 24.06.2019, KO, Shieli d., PF Akmaya, rice field edge and alfalfa fields, IT, AM. 


\section{Chrotogonus turanicus Kuthy, 1905}

Material examined. 5 males, 5 females, 17.05.2018, KO, neig. v. Abay, PF Akzhol, rice field edge, IT; 7 males, 9 females, 24-26.05.2018; KO, neig. v. Abay, PF Akzhol, rice field edge, IT; 2 males, 3 females; 24.06.2019, KO, Shieli d., PF Akmaya, rice field edge and alfalfa fields, IT, AM.

\section{Family Pamphagidae}

\section{Thrinchus arenosus (Bei-Bienko, 1948)}

Material examined. 2 males, 1 female, 24.05.2018, KO, neig. v. Abay, PF Akzhol, rice field edge, IT; 1 male, 1 female; 24.05.2019, KO, Shieli d., PF Akmaya, rice field edge and alfalfa fields, IT.

\section{Family Tetrigidae}

\section{Tetrix tartara (Saussure, 1887)}

Material examined. 11 males, 9 females, 17.05.2018, KO, neig. v. Abay, PF Akzhol, rice field edge, IT; 6 males, 7 females, 24.05.2018, KO, neig. v. Abay, PF Akzhol, rice field edge, IT; 4 males, 5 females, 24.06.2019, KO, Shieli d., PF Akmaya, rice field edge and alfalfa fields, IT, AM.

\section{Family Acrididae}

\section{Acrida oxycephala (Pallas, 1771)}

Material examined. 1 female, 12.07.2018, KO, Shieli d., PF Akmaya, rice field edge, IT; 1 male, 1 female, 22.06.2019, KO, neig. v. Abay, PF Akzhol, rice field edge and alfalfa fields, IT; 2 males, 1 female, 24.06.2019, KO, Shieli d., PF Akmaya, rice field edge and alfalfa fields, IT; 1 female, 30.08.2019, KO, Shieli d., PF Akmaya, rice field edge, IT.

\section{Acrotylus insubricus (Scopoli, 1786)}

Material examined. 4 males, 5 females, 17.05.2018, KO, neig. v. Abay, PF Akzhol, rice field edge, IT; 1 males, 1 female, 26.06.2018, KO, neig. v. Abay, PF Akzhol, rice field edge, IT; 7 males, 9 females, 12.07.2018, KO, Shieli d., PF Akmaya, rice field edge, IT, AM; 3 females, 23.04.2019, KO, Shieli d., PF Akmaya, at the hotel, manual collect, IT; 2 males, 3 females, 24.04.2019, KO, neig. v. Abay, PF Akzhol, rice field edge, IT. 


\section{Aiolopus thalassinus thalassinus (Fabricius, 1781)}

Material examined. 5 L5 males, 7 L5 females, 17.05.2018, KO, neig. v. Abay, PF Akzhol, rice field edge, IT; 8 L5 males, 5 L5 females, 24-26.05.2018, KO, neig. v. Abay, PF Akzhol, rice field edge, IT, AM; 4 males, 3 females, 24.06.2019, KO, Shieli d., PF Akmaya, rice field edge and alfalfa fields, IT, AM; 2 males, 1 female, 30.08.2019, KO, Shieli d., PF Akmaya, rice field edge, IT, AM.

\section{Aiolopus oxianus (Uvarov, 1926)}

Material examined. 8 L5 males, 6 L5 females, 17.05.2018, KO, neig. v. Abay, PF Akzhol, rice field edge, IT; 6 L5 males, 5 L5 females, 24-26.05.2018, KO, neig. v. Abay, PF Akzhol, rice field edge, IT, AM; 2 males, 1 female, 24.06.2019, KO, Shieli d., PF Akmaya, rice field edge and alfalfa fields, IT, AM; 2 female, 30.08.2019, KO, Shieli d., PF Akmaya, rice field edge, IT.

\section{Anacridium aegyptium (Linnaeus, 1758)}

Material examined. 2 males, 1 female, 17.05.2018, KO, neig. v. Abay, PF Akzhol, rice field edge, IT; 2 males, 3 females, 24.06.2019, KO, Shieli d., PF Akmaya, rice field edge, IT; 1 female, 30.08.2019, KO, Shieli d., PF Akmaya, rice field edge, IT.

\section{Calliptamus barbarus cephalotes Fischer von Waldheim, 1846}

Material examined. 4 L5 males, 7 L5 females, 17.05.2018, KO, neig. v. Abay, PF Akzhol, rice field edge, IT; 8 L5 males, 11 L5 females, 24-26.05.2018, KO, neig. v. Abay, PF Akzhol, rice field edge, IT, AM; 1 male, 1 female, 12.07.2018, KO, Shieli d., PF Akmaya, alfalfa fields, IT, AM; 4 males, 5 females, 13.07.2018, KO, Shieli d., PF Akmaya, to light, IT, AM; 2 males, 1 female, 24.06.2019, KO, Shieli d., PF Akmaya, rice field edge and alfalfa fields, IT, AM; 3 females, 30.08.2019, KO, Shieli d., PF Akmaya, rice field edge, IT.

\section{C. italicus (Linnaeus, 1758)}

Material examined. 5 L5 males, 6 L5 females, 17.05.2018, KO, neig. v. Abay, PF Akzhol, rice field edge, IT; 7 L5 males, 10 L5 females, 24-26.05.2018, KO, neig. v. Abay, PF Akzhol, rice field edge, IT, AM; 3 males, 4 females, 12.07.2018, KO, Shieli d., PF Akmaya, at the hotel, to the light, IT, AM; 2 males, 3 females, 13.07.2018, KO, Shieli d., PF Akmaya, alfalfa fields, IT; 3 males, 2 females, 30.08.2019, KO, Shieli d., 
PF Akmaya, rice field edge, IT, AM; 6 males, 7 females, 24.06.2019, KO, Shieli d., PF Akmaya, rice field edge and alfalfa fields, IT, AM. The conducted measurements of collected individuals both in 2018 and in 2019 showed that all exemplars belong to the single phase.

Celes variabilis (Pallas, 1774)

Material examined. 3 L5 males, 2 L5 females, 17.05.2018, KO, neig. v. Abay, PF Akzhol, rice field edge, IT; 5 males, 7 females, 24.06.2019, KO, Shieli d., PF Akmaya, rice field edge and alfalfa fields, IT, AM.

Distribution. Previously not listed in this area.

\section{Chrysochraon dispar (Germar, 1834)}

Material examined. 4 males, 6 females, 24.06.2019, KO, neig. v. Abay, PF Akzhol, rice field edge, IT, AM; 1 males, 1 female, 30.08.2019, KO, Shieli d., PF Akmaya, rice field edge, IT.

Distribution. Previously not listed in this area.

\section{Duroniella gracilis Uvarov, 1926}

Material examined. 4 L3, 4 L4, 2 L5, 1 female, 24-26.05.2018, KO, neig. v. Abay, PF Akzhol, rice field edge, IT, AM; 8 males, 11 females, 24.06.2019, KO, Shieli d., PF Akmaya, rice field edge and alfalfa fields, IT, AM.

\section{Duroniella kalmyka (Adelung, 1906)}

Material examined. 5 L3, 4 L4, 2 L5, 24-26.05.2018, KO, neig. v. Abay, PF Akzhol, rice field edge, IT; 1 males, 2 females, 24.06.2019, KO, Shieli d., PF Akmaya, rice field edge and alfalfa fields, IT, AM.

\section{Epacromius tergestinus (Charpentier, 1825)}

Material examined. 5 L5 males, 7 L5 우, 17.05.2018, KO, neig. v. Abay, PF Akzhol, rice field edge, IT; 8 L5 males, 7 L 5 females, 24-26.05.2018, KO, neig. v. Abay, PF Akzhol, rice field edge, IT, AM; 2 males, 5 females, 24.06.2019, KO, Shieli d., PF Akmaya, rice field edge and alfalfa fields, IT, AM; 2 males, 1 female, 30.08.2019, KO, Shieli d., PF Akmaya, rice field edge, IT, AM. 


\section{Eyprepocnemis unicolor Tarbinsky, 1928}

Material examined. 1 males, 1 female, 13.07.2018, KO, Shieli d., PF Akmaya, rice field edge, IT; 2 males, 2 females, 24.06.2019, KO, Shieli d., PF Akmaya, rice field edge and alfalfa fields, IT, AM.

\section{Gonista sagitta (Uvarov, 1912)}

Material examined. 2 L5 males, 1 L5 female, 17.05.2018, KO, neig. v. Abay, PF Akzhol, rice field edge, IT; 1 males, 24.06.2019, KO, Shieli d., PF Akmaya, alfalfa fields, IT; 1 female, 30.08.2019, KO, Shieli d., PF Akmaya, alfalfa fields, IT.

\section{Heteracris adspersa (Redtenbacher, 1889)}

Material examined. 3 males, 1 female, 30.08.2019, KO, Shieli d., PF Akmaya, rice field edge, IT, AM.

\section{Heteracris littoralis (Rambur, 1838)}

Material examined. 2 males, 3 females, 30.08.2019, KO, Shieli d., PF Akmaya, rice field edge and alfalfa fields, IT, AM.

\section{Heteracris pterosticha (Fischer von Waldheim, 1833)}

Material examined. 9 L2, 14 L3, 17.05.2018, KO, neig. v. Abay, PF Akzhol, rice field edge, IT; 8 L1, 11 L2, 5 L3, 4 L4, 2 L5, 24-26.05.2018, KO, neig. v. Abay, PF Akzhol, rice field edge, IT; 12 males, 11 females, 24.06.2019, KO, Shieli d., PF Akmaya, rice field edge and alfalfa fields, IT, AM.

\section{Hilethera turanica Uvarov, 1925}

Material examined. 1 female, 30.08.2019, KO, Shieli d., PF Akmaya, rice field edge, IT.

\section{Locusta migratoria (Linnaeus, 1758)}

Material examined. 22 L1, 15 L2, 14 L3, 17.05.2018, KO, neig. v. Abay, PF Akzhol, rice field edge, IT; 85 L1, 69 L2, 57 L3, 49 L4, 29 L5, 24-26.05.2018, KO, neig. v. 
Abay, PF Akzhol, rice field edge, IT, AM; 2 males, 3 females, 22.06.2019, KO, neig. v. Abay, PF Akzhol, rice field edge, IT, AM; 4 males, 5 females, 21.06.2019, KO, neig. v. Abay, PF Akzhol, rice field edge, IT, AM; 5 males, 3 females, 30.08.2019, KO, Shieli d., PF Akmaya, rice field edge, IT. In 2018, individuals of both the herd and transitional and solitary phases were present. The conducted measurements of individuals in 2019 showed that all exemplars belong to the single phase.

\section{Mecostethus parapleurus (Hagenbach, 1822)}

Material examined. 1 males, 1 female, 13.07.2018, KO, Shieli d., PF Akmaya, alfalfa fields, IT; 1 female, 21.06.2019, KO, neig. v. Abay, PF Akzhol, rice field edge, IT.

\section{Mesasippus kozhevnikovi (Tarbinsky, 1925)}

Material examined. 2 L 4, 2 L 5, 24.05.2019, KO, neig. v. Abay, PF Akzhol, rice field edge, IT; 1 males, 2 females, 21.06.2019, KO, neig. v. Abay, PF Akzhol, rice field edge, IT, AM; 1 males, 1 female, 22.06.2019, KO, neig. v. Abay, PF Akzhol, rice field edge, IT, AM; 3 males, 4 females, 24.06.2019, KO, Shieli d., PF Akmaya, rice field edge and alfalfa fields, IT, AM.

\section{Mioscirtus wagneri (Kittary, 1849)}

Material examined. 1 male, 1 female, 24.06.2019, KO, Shieli d., PF Akmaya, rice field edge and alfalfa fields, IT, AM.

\section{Oedipoda caerulescens (Linnaeus, 1758)}

Material examined. 5 L2, 4 L3, 17.05.2018, KO, neig. v. Abay, PF Akzhol, rice field edge, IT; 4 L4, 9 L5, 24-26.05.2018, KO, neig. v. Abay, PF Akzhol, rice field edge, IT, AM; 1 L5, 3 males, 2 females, 12.07.2018, KO, Shieli d., PF Akmaya, rice field edge and alfalfa fields, IT, AM.

\section{Oedipoda miniata (Pallas, 1771)}

Material examined. 4 L3, 17.05.2018, KO, neig. v. Abay, PF Akzhol, rice field edge, IT; 5 males, 4 females, 24.06.2019, KO, Shieli d., PF Akmaya, rice field edge and alfalfa fields, IT, AM. 


\section{Oxya fuscovittata (Marschall, 1836)}

Material examined. 12 L1, 10 L2, 14 L3, 17.05.2018, KO, neig. v. Abay, PF Akzhol, rice field edge, IT; 15 L2, 25 L3, 22 L4, 9 L5, 24-26.05.2018, KO, neig. v. Abay, PF Akzhol, rice field edge, IT, AM; 2 males, 3 females, 24.06.2019, KO, Shieli d., PF Akmaya, rice field edge, IT, AM.

\section{Pseudosphingonotus savignyi (Saussure, 1884)}

Material examined. 1 female, 24.05.2018, KO, neig. v. Abay, PF Akzhol, alfalfa field, IT; 1 males, 24.06.2019, KO, Shieli d., PF Akmaya, rice field edge and alfalfa fields, IT; 1 female, 27.05.2018, KO, neig. v. Abay, PF Akzhol, rice field edge, IT.

\section{Pyrgodera armata Fischer von Waldheim, 1846}

Material examined. 1 L2, 4 L3, 17.05.2018, KO, neig. v. Abay, PF Akzhol, rice field edge, IT; 4 L4, 2 L5, 24-26.05.2018, KO, neig. v. Abay, PF Akzhol, rice field edge, IT, AM; 1 L4, 27.05.2018, KO, neig. v. Abay, PF Akzhol, rice field edge, IT; 1 males, 2 females, 24.06.2019, KO, Shieli d., PF Akmaya, rice field edge, IT, AM.

\section{Sphingonotus rubescens (Walker, 1870)}

Material examined. 1 male, 17.05.2018, KO, neig. v. Abay, PF Akzhol, rice field edge, IT; 4 males, 5 females, 24.06.2019, KO, Shieli d., PF Akmaya, rice field edge, IT, AM.

\section{Tropidopola turanica turanica Uvarov, 1926}

Material examined. 4 L3, 17.05.2018, KO, neig. v. Abay, PF Akzhol, rice field edge, IT; 4 L4, 2 L5, 24-26.05.2018, KO, neig. v. Abay, PF Akzhol, rice field edge, IT, AM; 3 L5, 1 female, 30.08.2019, KO, Shieli d., PF Akmaya, rice field edge, IT, AM.

\section{Truxalis eximia Eichwald, 1830}

Material examined. 2 L4, 1 L5, 24-26.05.2018, KO, neig. v. Abay, PF Akzhol, rice field edge, IT; 1 female, 30.08.2019, KO, Shieli d., PF Akmaya, rice field edge, IT. 


\section{Discussion}

In general, only 21 families, 147 genera and 584 species of orthopteroid insects are currently known for the fauna of Kazakhstan. Of these, 2 families, 9 genera and 10 species of Mantids (Temreshev et al. 2015), 4 families, 11 genera and 20 species of Cockroaches (Bey-Bienko 1950; Temreshev et al. 2015; Temreshev 2017), 3 families, 6 genera and 7 species Earwigs (Bey-Bienko, 1936), 1 family, 1 genus and 2 species of Sticks insects (Temreshev et al. 2015), 11 families, 120 genera and 545 species of Orthopterans (Childebaev and Storozhenko 2001, 2004; Childebaev et al. 2014; Temreshev et al. 2015). Thus, 60 species from 58 genera and 17 families identified for the rice fields of the Kyzylorda area, providing a rather high percentage of the total number of orthopteroid insects in the Kazakhkhtan fauna.

For the rice fields of the Aral Sea M.Zh. Medetov (Medetov 2012) indicates a total of 12 species of Orthoptera (Katydids, True crickets, Pygmy grasshoppers, and True grasshoppers). In addition, according to O.S. Korsunovskaya (Korsunovskaya 2016), on the territory of Uzbekistan, a species of katydids Platycleis albopunctata transiens Zeuner, 1941 was noted, the finding of which is possible in the territory of the Kyzylorda region. For the vicinity of the Tasotkel reservoir in the Zhambyl oblast of Kazakhstan (Temreshev and Esenbekova 2017), 85 species of orthopteroid insects, belonging to 69 genera from 15 families and 5 orders, were identified first. Mantids (Mantodea) is represented by 3 families, 7 genera and 7 species; Cockroaches (Blattodea) - 2 families, 2 genera, 2 species; Earwigs (Dermaptera) - 3 families, 3 genera and 3 species; Sticks insects (Phasmoptera) - 1 family, 1 genus, 1 species; Orthopterans (Orthoptera) - 7 families, 55 genera and 72 species.

The relative richness of the fauna of orthopteroid insects in the studied areas of Kyzylorda compared with the Aral Sea region is explained by the great diversity of biotopes located in the vicinity of rice crops. There are numerous fields of various crops, wastelands, temporary and permanent living quarters, and natural stations clay semi-desert sections, reed beds, steppe stations, intrazonal sections along the banks of natural reservoirs and irrigation canals that allow the existence of a wide variety of species and life forms. From these sites insects periodically move, which are characteristic not only for rice agrocenosis. In addition, M.Zh. Medetov (Medetov 2012) for the Aral Sea region into account only Orthoptera, without Cockroaches, Mantids, Stick insects and Earwigs. For the vicinity of the Tasotkel reservoir (Temreshev and Esenbekova 2017), a greater variety of orthopteroid fauna was noted than for rice paddy crops in the Kyzylorda oblast. But it is located in a different climatic zone, characterized by a wide variety of natural biotopes and agrocenosis. Its entomofauna contains more northern elements.

\section{Conclusion}

In total, 60 species of orthopteroid insects belonging to 58 genera from 17 families and 5 orders were identified as a result of studies for agrocenosis of rice paddy fields 
in the Kyzylorda oblast of Kazakhstan. Mantids (Mantodea) are represented by 3 families (Empusidae, Mantidae, Tarachodidae), 6 genera and 6 species. Cockroaches (Blattodea) - 2 families (Polyphagidae, Blattidae), 2 genera, 2 species. Earwigs (Dermaptera) - 3 families (Labiduridae, Forficulidae, Spongiphoridae), 3 genera and 3 species. Sticks insects (Phasmoptera) - 1 family (Diapheromeridae), 1 genus, 1 species. The most numerous Orthoptera (Orthoptera) - 8 families, 46 genera and 48 species. Suborder Ensifera are represented by 3 families, including 9 genera and 11 species of Katydids (Tettigoniidae), 5 genera and 6 species of True crickets (Gryllidae), 1 genus and 1 species of Mole crickets (Gryllotalpidae). Suborder Caelifera - 5 families, including 1 genus and 1 species of Pygmy mole crickets (Tridactylidae), 2 genera and 2 species of Gaudy grasshoppers (Pyrgomorphidae), 1 genus and 1 species of Toad grasshoppers (Pamphagidae), 1 genus and 1 species of Pygmy grasshoppers (Tetrigidae), 27 genera and 30 species of real True grasshoppers (Acrididae). Of these, 3 species - B. brachyptera, $H$. tenuidentata and C. fuscipennis - are listed in the Red Book of the Republic of Kazakhstan. Two sinanthropic species were identified (S. lateralis and P. saussurei). Most of the species are economically harmful, of them C. variabilis and C. dispar previously not listed in this area, two are especially dangerous pests of agriculture (Italian locust $C$. italicus and Asian locust L. migratoria).

\section{Acknowledgements}

This work was carried out within the framework of the 019 budget programs "Introduction of a domestic biological product based on an entomopathogenic fungus against non-livestock locusts in the Kyzylorda oblast" and "Introduction on the basis of a pilot farm in the Kyzylorda oblast a complex of innovative agricultural technologies with elements of precision farming to increase the productivity of cultivated crops".

\section{References}

Beccaloni GW (2019) Cockroach Species File Online. Version 5.0/5.0. http://Cockroach. SpeciesFile.org

Bey-Bienko GYa (1936) Earwigs insects. Fauna of the USSR. A new series, 5. Moscow-Leningrad: Publishing House of the Academy of Sciences of the USSR. (In Russian).

Bey-Bienko GYa (1950) Cockroach insects. Fauna of the USSR. A new series, 40. MoscowLeningrad: Publishing House of the USSR Academy of Sciences. (In Russian).

Bey-Bienko GYa (1964a) Order Mantoptera (Mantodea, Mantoidea) - Mantis. In GYa BeyBienko (Eds.). Key of Insects of the European part of the USSR. I. Lower, ancient winged, with incomplete transformation. Moscow-Leningrad: Science. (In Russian).

Bey-Bienko GYa (1964b) Order Phasmoptera (Phasmoidea, Cheleutoptera) - Stick insects. In GYa Bey-Bienko (Eds.). Key of Insects of the European part of the USSR. I. Lower, ancient winged, with incomplete transformation. Moscow-Leningrad: Science. (In Russian). 
Bey-Bienko GYa (1964c) Order Orthoptera - Orthopterans. In GYa Bey-Bienko (Eds.). Key of Insects of the European part of the USSR. I. Lower, ancient winged, with incomplete transformation. Moscow-Leningrad: Science. (In Russian).

Brock PD, Büscher T, Baker E (2019) Phasmida Species File Online. Version5.0/5.0. Available from: http://Phasmida.SpeciesFile.org (Accessed: 11 July 2020).

Childebaev MK, Storozhenko SYu (2001) An annotated list of brachycerous orthopterous insects (Orthoptera: Caelifera) occurring in Kazakhstan. Tethys Entomological Research, 3: 15-60.

Childebaev MK, Storozhenko SYu (2004) An annotated list of the long-horned orthopterans (Orthoptera: Ensifera) of Kazakhstan. Tethys Entomological Research, 9: 213-228.

Childebaev MK, Temreshev II, Kolov CV (2014) Myrmecophilus acervorum (Panzer, 1799) (Orthoptera, Myrmecophilidae) - first records ant-loving cricket for the fauna of Kazakhstan. Euroasian entomological journal 13 (3): 246. (In Russian).

Cigliano MM, Braun H, Eades DC, Otte D (2019) Orthoptera Species File Online. Version 5.0/5.0. Available from: http://Orthoptera.SpeciesFile.org (Accessed: 11 July 2020).

Hopkins H, Maehr MD, Haas F, Deem LS (2019) Dermaptera Species File. Version 5.0/5.0. Available from: http://Dermaptera.SpeciesFile.org (Accessed: 11 July 2020).

Jacobson GG and Bianchi VL (1905) Orthopteroid and Pseudoneuropteroid insects of Russian Empire and adjacent countries. SPb.: Devrien Publ. (In Russian).

Kotlyarova LA (1975) Rice pests in southern Kazakhstan and control measures: Abstract. diss. Cand. of agricultural sciences. Alma-Ata. (In Russian).

Komissarova IA (1973) Rice pests in the new rice growing zone. Agricultural Bulletin. Science of Kazakhstan 8: 102-104. (In Russian).

Korsunovskaya OS (2016) On the Distribution of the Bush Cricket Platycleis albopunctata transiens Zeuner, 1941 (Orthoptera, Tettigoniidae) in the South of European Russia and in Uzbekistan. Entomological Review 96 (3): 288-293.

Latchininsky AV, Sergeev MG, Childebaev MK, Chernyakhovskiy ME, Lockwood JA, Kambulin VE, Gapparov FA (2002) Locusts of Kazakhstan, Central Asia and adjacent territories. Laramie: International Association of Applied acridology and University of Wyoming. (In Russian).

Medetov MZh (2012) Fauna and formation (Insecta: Orthoptera) of Southern at Aral. Abstract. diss. Cand. of biological sciences. Tashkent. (In Russian).

Otte D, Spearman L, Stiewe MBD, Eades DC (2019) Mantodea Species File Online. Version 5.0/5.0. Available from: http://Mantodea.SpeciesFile.org (Accessed: 11 July 2020).

Pravdin FN (1978) Ecological geography of insects of Central Asia. Orthopteroids. Moscow: Nauka. (In Russian).

Sergeev MG, Childebaev MK, Vankova IA, Gapparov FA, Kambulin VE, Kokanova E, Lachininsky AV, Pshenitsyna LB, Temreshev II, Chernyakhovsky ME, Sobolev NN, Molodtsov VV (2016) Italian locust [Calliptamus italicus (Linnaeus, 1758)]: morphology, distribution, ecology, population management / Ed. M.G. Sergeev and M.K. Childebaev. Rome: Food and Agriculture Organization of the United Nations. (In Russian). 
Shcherbakov EO, Savitsky VYu (2015) New Data on the Fauna, Taxonomy and Ecology of Praying Mantises (Dictyoptera, Mantodea) from Russia. Entomological Review 95 (2): 181-199.

Storozhenko SYu (2004) Long-horned orthopterans (Orthoptera: Ensifera) of the Asian part of Russia. Vladivostok: Dalnauka. (In Russian).

Temreshev II (2017) Pests of stocks and raw materials, common in the territory of the Republic of Kazakhstan, and some related and quarantine species (species composition and brief technology protection measures). Second edition, revised and supplemented. Almaty: LLP "Nur-Print" (In Russian).

Temreshev II, Esenbekova PA (2017) Ortopteroid insects (Insecta, Orthopteroidea) of the Tasotkel water reservoir Zambyl area (Kazakhstan). Acta Biologica Sibirica 3 (1): $13-$ 22. (In Russian). https://doi.org/10.14258/abs.v3i1.2178

Temreshev II, Kazenas VL, Childebaev MK, Isenova GZh, Kozabaeva GE (2015) A preliminary list of indicator species of insects in South Kazakhstan. Almaty: Nur-Print (In Russian). 